OPEN ACCESS

Edited by:

Jun Yan,

Harbin Engineering University, China

Reviewed by:

Hao Chen,

Zhejiang Agriculture and Forestry

University, China

Shulan Wang,

Northeastern University, China

Hongying Quan,

Nanchang Hangkong University, China

*Correspondence:

Tianyu Liu

tliu23@vt.edu

Specialty section:

This article was submitted to Electrochemical Energy Conversion

and Storage,

a section of the journal

Frontiers in Energy Research

Received: 29 April 2020

Accepted: 25 May 2020

Published: 23 June 2020

Citation:

Liu T (2020) Overlooking Issues and Prospective Resolutions Behind the

Prosperity of Three-Dimensional

Porous Carbon Supercapacitor

Electrodes. Front. Energy Res. 8:125.

doi: 10.3389/fenrg.2020.00125

\section{Overlooking Issues and Prospective Resolutions Behind the Prosperity of Three-Dimensional Porous Carbon Supercapacitor Electrodes}

\author{
Tianyu Liu* \\ Department of Chemistry, Virginia Polytechnic Institute and State University, Blacksburg, VA, United States
}

The past decade has witnessed the boom of porous carbon materials, especially three-dimensional (3D) ones, as supercapacitor electrode materials. Their large surface areas, tunable pore volumes, adjustable degrees of pore interconnectivity, and the potential to possess hierarchical porous networks have enabled them to achieve simultaneously high capacitance and excellent rate capability. This feature is challenging for non-3D counterparts but favorable for supercapacitors. However, behind the prosperity come the problems, and unfortunately, most of the issues are left unacknowledged and unaddressed in the literature available. This perspective aims to identify these problems, discuss the reasons for overlooking, and provide possible solutions to address these issues. The author wishes this article will draw the attention of researchers in electrochemical energy storage to tackle these roadblocks in the future development of 3D porous carbon electrodes in supercapacitors.

Keywords: three-dimensional, pore, carbon, supercapacitor, electrodes, overlooking issue

\section{INTRODUCTION}

Accompanying with the boom of electrochemical energy storage, three-dimensional (3D) porous carbon materials have emerged as rising stars of electrodes in supercapacitors. Supercapacitors, including electrical double-layer capacitors and pseudocapacitors, are electrochemical energy storage devices with power density typically about 100 times higher than those of rechargeable batteries. Their high power density bestows their applications in ultrafast charging and discharging scenarios (Miller and Simon, 2008; Salunkhe et al., 2014). As indispensable components in supercapacitors, electrodes having ultrasmall electrical resistance and ultrahigh surface areas are preferred. Therefore, a diverse array of highly conductive carbon materials with tunable morphologies have become supercapacitor electrode candidates for decades.

One of the representative conventional supercapacitor carbon electrodes is activated carbon. These carbon particles possess abundant micropores and surface areas surpassing $1,000 \mathrm{~m}^{2}$ $\mathrm{g}^{-1}$. They store charges mainly via the formation of electrical double layers and are used in electrical double-layer capacitors (Ji et al., 2014) or capacitive electrodes in supercapacitor-battery hybrids (Liu H. et al., 2020). Activated carbon, however, is far from ideal. Its disadvantage is two-folded. On the one hand, its powdered form demands polymer binders when being processed into electrodes, which introduces unnecessary internal resistance born from the particle-particle contact resistance as well as the low electrical conductivity of the binders (Liu T. et al., 2020). On the other hand, the micropore-dominated porous structure of activated 
carbon impedes ion diffusion throughout a particle, limiting the pore accessibility and, subsequently, the capacitance and energy density (Zhang et al., 2017). These two drawbacks make activated carbon unsuitable for storing large amounts of charges at elevated charge and discharge rates, the conditions under which supercapacitors are supposed to work.

The shortcomings of conventional powdered porous carbon materials have motivated the development of three-dimensional porous carbons as supercapacitor electrodes within the past decade. 3D porous carbons with high surface areas and easy ionaccessible pores allow capacitance and remarkable rate capability mutually achievable (Liu et al., 2017). Besides, the structural and morphological diversity and tunability derived from the versatility in sources and synthesis processes have generated a tremendous number of $3 \mathrm{D}$ carbon supercapacitor electrodes in the literature (Zhu et al., 2016; Zhang et al., 2017, 2019; Yang et al., 2019). The variety in structural parameters, including surface areas, pore interconnectivity, pore volumes, pore size distributions, and surface functionalities, is far more significant than those of non-3D carbon counterparts. These characteristics have also pushed the capacitance of carbon materials to beyond $300 \mathrm{~F} \mathrm{~g}^{-1}$ (Liu et al., 2017), a level unattainable by conventional porous carbon materials such as activated carbon.

The thrive of $3 \mathrm{D}$ porous carbon materials, however, brings about issues that compromise the scientific quality and integrity of relevant works. Unfortunately, most of the problems have been left unacknowledged and unaddressed (Figure 1). Without exhaustively examining the literature, this perspective aims to analyze these critical issues that the author hopes to initiate discussions and cultivate consensuses among the researchers in the field of electrochemical energy storage. Corresponding solutions are given in each section below.

\section{COMPRESSING DAMAGES 3D STRUCTURE}

Mechanical pressing or calendering, which improve the contact between carbon materials and their current collectors, are needed for manufacturing supercapacitor electrodes. These processes not only reduce the contact resistance of supercapacitor electrodes (Dsoke et al., 2013), but also increase the volumetric performance metrics such as volumetric capacitance, energy density, and power density, due to volumetric densification. As a result, most researchers mechanically compress their as-prepared 3D carbon materials onto conductive substrates, e.g., nickel foam (Zhang et al., 2017) and aluminum foil (Shah et al., 2020). In some cases, $3 \mathrm{D}$ porous carbon materials are ground into powders, blended with binders and carbon black (electrically conductive additive) when making electrodes (Deng et al., 2019; Nawwar et al., 2020). Inevitably, the delicate 3D architectures of the active materials are substantially altered, if not entirely demolished, after compression or grinding. Therefore, claiming that hierarchical porous structures facilitate ion diffusion or render outstanding rate capability based on morphologies before compression or grinding is inappropriate to reflect the actual working conditions of $3 \mathrm{D}$ porous carbon materials.
The most straightforward resolution is to avoid compressing or grinding $3 \mathrm{D}$ porous carbon materials. For example, Zhu et al. attached compressed $\mathrm{Ni}$ foam onto 3D-printed graphene aerogels using silver paste (Zhu et al., 2016), and Zhai et al. soldered copper foil onto $\mathrm{Ni}$-foam-templated graphene foam (Zhai et al., 2015), both of which preserved the 3D morphologies of the active materials. However, silver or solder are prone to oxidation under positive biases, giving rise to unwanted oxidation current and redox peaks. These problems require proper sealing of the contact points (e.g., by epoxy) that complicates the fabrication, adds electrode dead mass, and leads to substantial performance deviations due to the variation in the sealant coverage. Additionally, the contact resistance might be large or small, depending on the adhesion strength between the electrodes and the current collectors. This factor makes the electrochemical performance irreproducible.

A relatively simple solution is to acknowledge the necessity of compression, but provide comprehensive characterizations to support discussions. Probing the morphologies of compressed $3 \mathrm{D}$ porous carbon materials is necessary. Based on the author's experience, compression only shrinks macropores. Still, it preserves mesopores and micropores, so the surface area of a $3 \mathrm{D}$ porous carbon material will unlikely decrease significantly compared to its uncompressed form. If macropores are partially maintained, the $3 \mathrm{D}$ porous network of the carbon material is still retained, though the morphology is different. Grinding, on the contrary, will most likely destroy 3D architectures entirely to broken particles and hence, is not recommended for processing $3 \mathrm{D}$ porous carbon materials. Top-view and cross-sectional-view scanning electron microscopy images are critical to illustrate the compressed morphologies of the active materials. Characterizing the compressed carbon materials with gas physisorption is imperative to gauge any subtle changes in the surface areas and pore size distributions. Both techniques provide foundations for discussing the structure-performance relationship of $3 \mathrm{D}$ porous carbon electrodes. For readers to gain comprehensive understandings of the reported materials, supplementing typical characterization results of as-prepared 3D porous carbon materials with those of compressed counterparts is encouraged.

\section{TO BE OR NOT TO BE SELF-STANDING?}

Self-standing has emerged as a fashion term to describe the feature of $3 \mathrm{D}$ porous carbon electrodes. However, arbitrarily using this word without considering the characteristic of the electrodes can lead to false and misleading information.

The difference between "self-standing material" and "selfstanding electrode" must be strictly distinguished. Monolithic $3 \mathrm{D}$ porous carbon materials should be self-standing by nature, but whether they constitute self-standing electrodes depend on the fabrication method. If binders are used to bind 3D carbon materials or their grounded granules when making electrodes, such binder-containing electrodes can no longer be called self-standing. Rigorously speaking, electrodes are not self-standing if current collectors are used; however, 

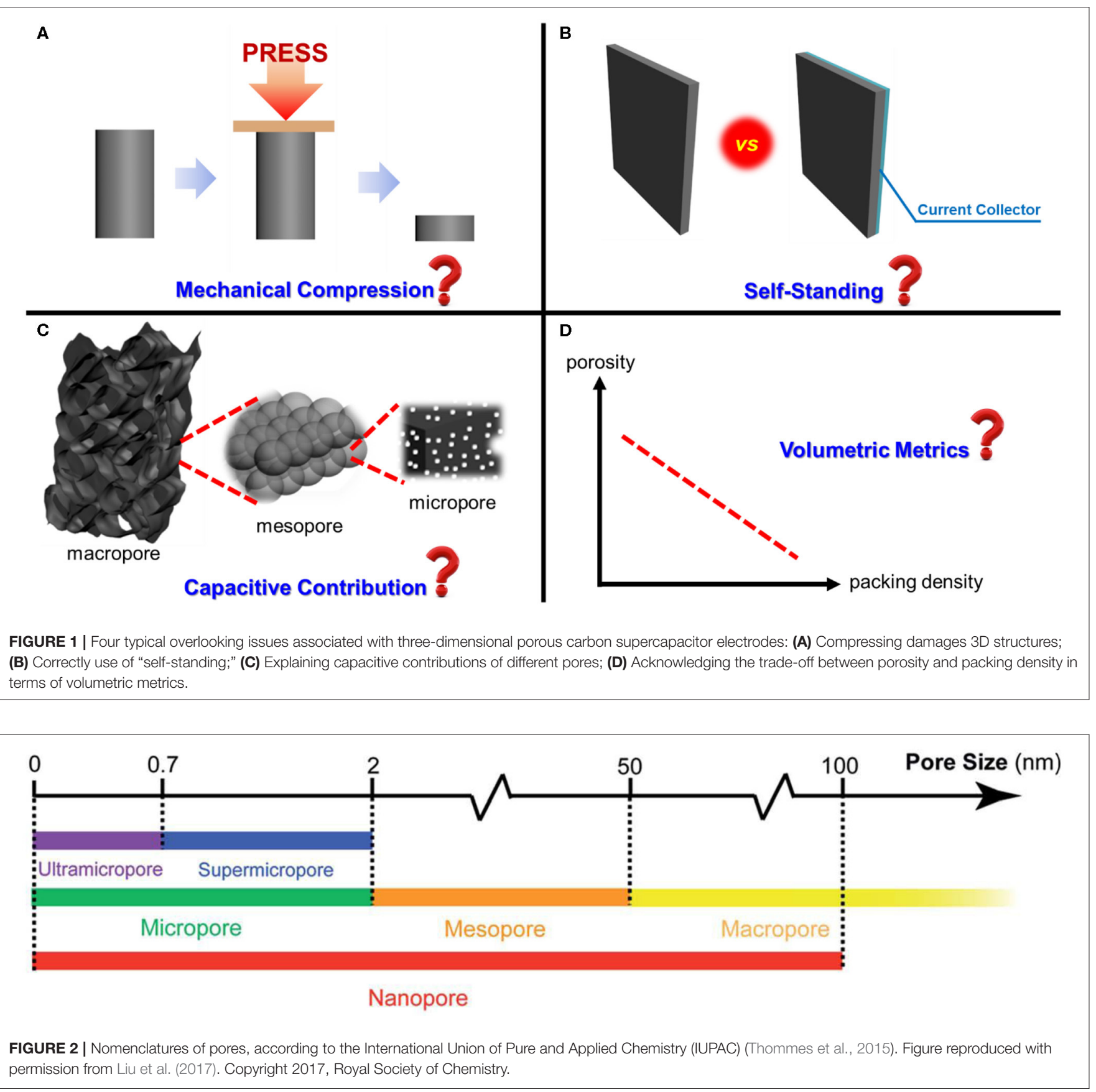

the author believes that removing current collectors will create unnecessary technical challenges to the evaluation of supercapacitor electrodes, so calling binder-free electrodes selfstanding is acceptable.

This issue calls for the transparency of experimental conditions. Authors reporting 3D carbon electrodes in supercapacitors or electrochemical energy storage, if broadly speaking, need to present explicitly how the electrodes are made. These conditions include the use of current collectors, compositions and manufacturers of current collectors, how carbon materials are adhered to current collectors (e.g., use of binders or direct compressing), as well as the mass loadings of active materials. The bottom line is that any researchers should be able to reproduce the experiments following the provided technical details with absolutely no confusion.

\section{ELUCIDATING PORE CONTRIBUTIONS}

One of the unique structural features of $3 \mathrm{D}$ porous carbon materials is their hierarchically porous structures, that is, 
structures with interconnecting pores of different sizes (Figure 2) assembled into hierarchical networks. Porous materials that contain multiscale but spatially isolated pores are not hierarchical, because their pores cannot "communicate" with each other during charge and discharge processes. Due to the diversified pore sizes and shapes, differentiating the capacitive contributions of the pores becomes increasingly critical to rationalize the design of next-generation porous carbon supercapacitor electrodes.

Despite extensive investigations, the contributions of different pores have yet reached an agreement. The general belief is that macropores and mesopores contribute little to capacitance, but they help electrolyte infiltration and facilitate ion diffusion, both of which improve the ion accessibility to micropores (Yang et al., 2017). Zhang et al. have experimentally verified that sub-micron macropores and mesopores in a chitosan-derived carbon aerogel are indispensable for maintaining its excellent rate capability (Zhang et al., 2017). Micropores are mainly responsible for high surface areas and ion storage, resulting in capacitance. The general belief, however, is challenged by simulation works that show macropores and mesopores are unnecessary, as they facilitate electrolyte infiltration but not ion diffusion (Borchardt et al., 2018). Others have revealed that micropores of pore openings less than $1 \mathrm{~nm}$ can accelerate ion diffusion due to the ion packing patterns in ionophobic pores (Mo et al., 2020). Although the author is puzzled about the exact causes of the discrepancy between the simulation results and experimental observations, a possible reason is the different experimental setups. Most simulations choose ionic liquids as study objects because of their simplicity (no solvent molecules and ion solvation) to reduce calculation costs. In contrast, the majority of laboratory supercapacitors adopt aqueous electrolytes because of their safety, ease of fabrication, and intrinsically rapid ion diffusion.

Besides simulations, some experimental observations also contradict the general belief. For example, depending on the source, commercial activated carbon can contain mesopores slightly bigger than $2 \mathrm{~nm}$, according to $\mathrm{N}_{2}$-physisorption (Liu T. et al., 2020), but their capacitance still decreases dramatically at elevated discharging rates. Carbide-derived carbons, though possessing large numbers of mesopores and macropores, still exhibit satisfactory rate capability in aqueous electrolytes (Huang et al., 2016).

These outliers in the literature highlight the complex roles of various pores. Perhaps the pore geometry, surface functionality, and graphitization degrees of $3 \mathrm{D}$ porous carbon electrodes all profoundly alter the electrochemical responses. For example, Dyatkin et al. simulated that the oxygen functional groups inside pores attracted planar anions in ionic liquids. This attraction aligned the anions in parallel to the pore surface and provided relatively clear transport channels to enhance rate capability (Dyatkin et al., 2016). The alliance between experimentalists and theorists, as well as alignment between experimental conditions and computational inputs, are undoubtedly indispensable to drive future investigations on the charge-storage mechanisms of 3D porous carbon electrodes. Additionally, the progress of advanced characterization methods, including quasi-elastic neutron scattering (Osti and Mamontov, 2020), quartz crystal microbalance (Levi et al., 2016), in-situ nuclear magnetic resonance spectroscopy (Forse et al., 2017), are vital tools for future investigations. Supporting experimental findings with simulation results in a research article is strongly encouraged if condition permits. This practice may become a new norm for publishing manuscripts in top-tier journals and can reward interdisciplinary researches with crystal-clear mutual communications among all the practitioners involved. The author believes that the role-determination of pores in versatile $3 \mathrm{D}$ porous carbon materials will remain as frontiers in electrochemical energy storage.

\section{PACKING DENSITY VS. POROSITY}

Perhaps the most common criticism of 3D porous carbon electrodes is their large porosity that declines the packing density of an electrode. Small packing density leads to low volumetric performance metrics, such as volumetric capacitance.

While the author fully agrees that volumetric metrics are crucial for rechargeable batteries whose working space is tight (Choi and Aurbach, 2016), the working environments of supercapacitors are far more diversified than those of batteries. $3 \mathrm{D}$ porous carbon materials with large porosity have the advantage of low volumetric density or lightweight. This characteristic makes them especially suitable for portable electronics needing high power density, such as drills and drones (Lin et al., 2018). In such cases, the volume of an energystorage device is less considerable than its weight. Therefore, the author believes that it is unfair to criticize $3 \mathrm{D}$ porous carbon electrodes based on their low packing density. Comprehensive evaluations of a 3D porous carbon electrode and reporting a full set of gravimetric, areal, and volumetric performance metrics are highly recommended.

Another issue calling attention is the report of the gravimetric capacitance of $3 \mathrm{D}$ porous carbon materials. The relationship between the total capacitance $\left(C_{T}\right)$ and gravimetric capacitance, or specific capacitance $\left(C_{m}\right)$, of an electrode, is:

$$
C_{T}=C_{m} \times m
$$

where $m$ is the mass of active materials. Based on this equation, achieving ultrahigh numbers of gravimetric capacitance becomes diminishingly meaningful if the mass loading of the active material is limited, as the total capacity is insignificant. Therefore, it is always a good practice to report gravimetric performance in the context of mass loading. For commercial viability, the mass loading of an electrode material must be $10 \mathrm{mg} \mathrm{cm}^{-2}$ or above (Chen et al., 2020). Still, this standard should not be rigid to restrict the development of laboratory-scale supercapacitors, which usually involve mass loadings of active materials between 1 and $10 \mathrm{mg} \mathrm{cm}^{-2}$. Nevertheless, explicitly reporting mass loading and accurately characterizing the influence of mass loading on electrochemical performance are always welcome and practically meaningful (Lin et al., 2015). Additionally, as areal capacitance depends on mass loading, reporting both gravimetric capacitance 
and areal capacitance for single electrodes is beneficial to avoid deceitfully excellent charge-storage performance.

\section{CONCLUSIONS AND OUTLOOKS}

This perspective highlights four main issues behind the prosperity of $3 \mathrm{D}$ porous carbon materials as supercapacitor electrodes. They are mostly overlooked in the existing literature, and if continually left unaddressed, are harmful to the healthy development of supercapacitors. Hence, the article presents the current understanding, points out the existing challenges, and discusses the possible resolutions of each issue. The author hopes this perspective will draw the attention of the practitioners in the

\section{REFERENCES}

Borchardt, L., Leistenschneider, D., Haase, J., and Dvoyashkin, M. (2018). Revising the concept of pore hierarchy for ionic transport in carbon materials for supercapacitors. Adv. Energy Mater. 8:1800892. doi: 10.1002/ aenm.201800892

Chen, R., Yu, M., Sahu, R. P., Puri, I. K., and Zhitomirsky, I. (2020). The development of pseudocapacitor electrodes and devices with high active mass loading. Adv. Energy Mater. 10:1903848. doi: 10.1002/ aenm. 201903848

Choi, J. W., and Aurbach, D. (2016). Promise and reality of post-lithiumion batteries with high energy densities. Nat. Rev. Mater. 1:16013. doi: $10.1038 /$ natrevmats.2016.13

Deng, X., Zhua, S., Li, J., He, F., Liu, E., He, C., et al. (2019). Bio-inspired three-dimensional carbon network with enhanced mass-transfer ability for supercapacitors. Carbon 143, 728-735. doi: 10.1016/j.carbon.2018.11.055

Dsoke, S., Tian, X., Täubert, C., Schlüter, S., and Wohlfahrt-Mehrens, M. (2013). Strategies to reduce the resistance sources on electrochemical double layer capacitor electrodes. J. Power Sources 238, 422-429. doi: 10.1016/j.jpowsour.2013.04.031

Dyatkin, B., Zhang, Y., Mamontov, E., Kolesnikov, A. I., Cheng, Y., Meyer, H. M., et al. (2016). Influence of surface oxidation onion dynamics and capacitance in porous and nonporous carbon electrodes. J. Phys. Chem. C 120, 8730-8741. doi: 10.1021/acs.jpcc.6b01204

Forse, A. C., Griffin, J. M., Merlet, C., Carretero-Gonzalez, J., Raji, A.-R. O., Trease, N. M., et al. (2017). Direct observation of ion dynamics in supercapacitor electrodes using in situ diffusion NMR spectroscopy. Nat Energy 2:16216. doi: 10.1038/nenergy.2016.216

Huang, P., Lethien, C., Pinaud, S., Brousse, K., Laloo, R., Turq, V., et al. (2016). Onchip and freestanding elastic carbon films for micro-supercapacitors. Science 351, 691-695. doi: 10.1126/science.aad3345

Ji, H., Zhao, X., Qiao, Z., Jung, J., Zhu, Y., Lu, Y., et al. (2014). Capacitance of carbon-based electrical double-layer capacitors. Nat. Commun. 5:3317. doi: $10.1038 /$ ncomms4317

Levi, M. D., Daikhin, L., Aurbach, D., and Presser, V. (2016). Quartz crystal microbalance with dissipation monitoring (EQCM-D) for in-situ studies of electrodes for supercapacitors and batteries: a mini-review. Electrochem. Commun. 67, 16-21. doi: 10.1016/j.elecom.2016.03.006

Lin, T., Chen, I. W., Liu, F., Yang, C., Bi, H., Xu, F., et al. (2015). Nitrogen-doped mesoporous carbon of extraordinary capacitance for electrochemical energy storage. Science 350, 1508-1513. doi: 10.1126/science.aab3798

Lin, Z., Goikolea, E., Balducci, A., Naoi, K., Taberna, P. L., Salanne, M. G., et al. (2018). Materials for supercapacitors: When Li-ion battery power is not enough. Mater. Today 21, 419-436. doi: 10.1016/j.mattod.2018.01.035

Liu, H., Liu, X., Wang, S., Liu, H.-K., and Li, L. (2020). Transition metal based battery-type electrodes in hybrid supercapacitors: a review. Energy Stor. Mater. 28, 122-145. doi: 10.1016/j.ensm.2020.03.003

Liu, T., Serrano, J., Elliott, J., Yang, X., Cathcart, W., Wang, Z., et al. (2020). Exceptional capacitive deionization rate and capacity by block electrochemical energy storage community to address the issues by intimate collaboration, protocol unification, and frequent idea exchange in the ensuing years.

\section{AUTHOR CONTRIBUTIONS}

The author confirms being the sole contributor of this work and has approved it for publication.

\section{ACKNOWLEDGMENTS}

We acknowledges Dr. Francesco Coneri for his assistance in arranging the submission of this article.

copolymer-based porous carbon fibers. Sci. Adv. 6:eaaz0906. doi: 10.1126/ sciadv.aaz0906

Liu, T., Zhang, F., Song, Y., and Li, Y. (2017). Revitalizing carbon supercapacitor electrodes with hierarchical porous structures. J. Mater. Chem. A 5, 17151-17173. doi: $10.1039 / \mathrm{c} 7 \mathrm{ta} 05646 \mathrm{j}$

Miller, J. R., and Simon, P. (2008). Materials science. Electrochemical capacitors for energy management. Science 321, 651-652. doi: 10.1126/ science. 1158736

Mo, T., Bi, S., Zhang, Y., Presser, V., Wang, X., Gogotsi, Y., et al. (2020). Ion structure transition enhances charging dynamics in subnanometer pores. ACS Nano 14, 2395-2403. doi: 10.1021/acsnano.9b09648

Nawwar, M., Sahu, R. P., Puri, I. K., and Zhitomirsky, I. (2020). Functionally decorated carbon nanotube networks for energy storage in supercapacitors. Front. Energy Res. 8:46. doi: 10.3389/fenrg.2020.00046

Osti, N. C., and Mamontov, E. (2020). Microscopic dynamics in room-temperature ionic liquids confined in materials for supercapacitor applications Sust. Energy Fuels 4, 1554-1575. doi: 10.1039/C9SE00829B

Salunkhe, R. R., Lee, Y. H., Chang, K. H., Li, J. M., Simon, P., Tang, J., et al. (2014). Nanoarchitectured graphene-based supercapacitors for next-generation energy-storage applications. Chemistry 20, 13838-13852. doi: 10.1002/chem.201403649

Shah, S. A., Kulhanek, D., Sun, W., Zhao, X., Yu, S., Parviz, D., et al. (2020). Aramid nanofiber-reinforced three-dimensional graphene hydrogels for supercapacitor electrodes. J. Colloid Interface Sci. 560, 581-588. doi: 10.1016/j.jcis. 2019.10.066

Thommes, M., Kaneko, K., Neimark, A. V., Olivier, J. P., Rodriguez-Reinoso, F., Rouquerol, J., et al. (2015). Physisorption of gases, with special reference to the evaluation of surface area and pore size distribution (IUPAC Technical Report). Pure Appl. Chem. 87, 1051-1069. doi: 10.1515/pac-2014-1117

Yang, J., Wu, H., Zhu, M., Ren, W., Lin, Y., Chen, H., et al. (2017). Optimized mesopores enabling enhanced rate performance in novel ultrahigh surface area meso-/microporous carbon for supercapacitors. Nano Energy 33, 453-461. doi: 10.1016/j.nanoen.2017.02.007

Yang, S., Wang, S., Liu, X., and Li, L. (2019). Biomass derived interconnected hierarchical micro-meso-macro- porous carbon with ultrahigh capacitance for supercapacitors. Carbon 147, 540-549. doi: 10.1016/j.carbon.2019.03.023

Zhai, T., Lu, X., Wang, H., Wang, G., Mathis, T., Liu, T., et al. (2015). An electrochemical capacitor with applicable energy density of $7.4 \mathrm{Wh} / \mathrm{kg}$ at average power density of $3000 \mathrm{~W} / \mathrm{kg}$. Nano Lett. 15, 3189-3194. doi: 10.1021/acs.nanolett.5b00321

Zhang, F., Liu, T., Li, M., Yu, M., Luo, Y., Tong, Y., et al. (2017). Multiscale pore network boosts capacitance of carbon electrodes for ultrafast charging. Nano Lett. 17, 3097-3104. doi: 10.1021/acs.nanolett.7b00533

Zhang, Y., Yang, S., Wang, S., Liu, X., and Li, L. (2019). Microwave/freeze casting assisted fabrication of carbon frameworks derived from embedded upholder in tremella for superior performance supercapacitors. Energy Storage Mater. 18, 447-455. doi: 10.1016/j.ensm.2018.08.006

Zhu, C., Liu, T., Qian, F., Han, Y.-J., Duoss, E. B., Kuntz, J. D., et al. (2016). Supercapacitors based on three-dimensional hierarchical 
graphene aerogels with periodic macropores. Nano Lett. 16, 3448-3456. doi: 10.1021/acs.nanolett.5b04965

Conflict of Interest: The author declares that the research was conducted in the absence of any commercial or financial relationships that could be construed as a potential conflict of interest.
Copyright $\odot 2020$ Liu. This is an open-access article distributed under the terms of the Creative Commons Attribution License (CC BY). The use, distribution or reproduction in other forums is permitted, provided the original author(s) and the copyright owner(s) are credited and that the original publication in this journal is cited, in accordance with accepted academic practice. No use, distribution or reproduction is permitted which does not comply with these terms. 